\title{
Using crystallographic models to experimentally measure partial atomic charges with comparison to quantum calculations
}

\author{
T Keller ${ }^{1}$, M Zdilla $^{2}$, P Prakash ${ }^{3}$, A Byrne ${ }^{1}$ \\ ${ }^{1}$ Temple University, Philadelphia, PA, ${ }^{2}$ Chemistry, Temple Univ, Philadelphia, PA, ${ }^{3}$ IISER Pune, \\ Pune, Maharashtra \\ tmkeller@temple.edu
}

Modern crystallographic refinement methods treat each atom in a molecule as neutral with spherical electron density. Atoms, however, exhibit partial atomic charges arising from intramolecular forces via bonding. These partial charges are crucial for understanding electronic structure and bulk physical properties of molecules. Typically the polarity and polarizability of molecules are calculated using IR and Raman spectroscopy, respectively. While these techniques can be used on small molecules, fine elucidation of partial charges on individual atoms is still unrealized. Here we present crystallographic refinement developments that allow us to refine electron density around individual atoms to experimentally calculate partial atomic charges. Comparison between these experimentally calculated charges to theoretical quantum calculated charges will also be presented.

Acta Cryst. (2020). A76, a193 